\title{
Gravitational Lensing of Spiral Vortex Solar Radiation by Venus
}

\author{
Genrik A. Nikolsky' ${ }^{1}$ Alexander F. Pugach² \\ ${ }^{1}$ Physical Department, St. Petersburg University, St. Petersburg, Russia \\ ${ }^{2}$ Main Astronomical Observatory of NAS of Ukraine, Kiev, Ukraine \\ Email: gnik777@mail.ru,pugach@yandex.ru,pugach@mao.kiev.ua
}

Received 11 May 2016; accepted 1 August 2016; published 4 August 2016

Copyright (C) 2016 by authors and OALib.

This work is licensed under the Creative Commons Attribution International License (CC BY). http://creativecommons.org/licenses/by/4.0/

(c) (i) Open Access

\section{Abstract}

Instrumental observations of the Venus transit on June 6, 2012 were performed using two similar torsinds-a special kind of ultralight torsion balances on a silk suspension. A clear response of both devices to this phenomenon was registered. The observations have verified a theoretical assumption on existence of the Spiral Vortex Solar Radiation (SVSR). The direct measurements of a space velocity of the SVSR determined on the track Venus-Earth, gave a value of $1990 \mathrm{~km} / \mathrm{s}$ which coincided almost exactly with the previously theoretical calculations. Given the insensitivity of the torsind to gravitational and electromagnetic influences a hypothesis is suggested that the observation manifests a new fundamental interaction.

\section{Keywords}

Spiral Vortex Solar Radiation, Torsion Indicators, Transit of Venus, Gravitational Lensing

Subject Areas: Experimental Physics

\section{Introduction}

The first experimental studies using a variety of sensitive torsion indicators were conducted at the beginning of the XX century. These results suggest the existence of a vortex field or a torsion field, which is grounded in the advanced physics of Einstein-Cartan theory. Sun is suggested to be a credible source of such a field.

Apparently, the first professional researcher of a torsion phenomenon was a professor of Novo-Alexandria Academy Meteorological Observatory N. P. Myshkin [1]. He conducted research with a torsion radiometer which consisted of a round mica plate horizontally suspended on a bifilar thin silk thread inside a wood/glass housing.

As a result of long-term experiments Myshkin has found that the plate came to right- or left-handed rotation, depending on the intensity of sunlight both direct and scattered as well as under the influence of meteorological 
and astronomical conditions and the time of day.

Based on this, professor Myshkin concluded that the origin of the rotational force has no relation neither to gravity nor magnetism. The author excluded the convective air movement inside his device as a cause of revolution of the suspended mica plate. The main conclusion of these studies was the suspicion on the existence of an unknown force field which swirls the mobile part of the radiometer to the left or the right.

The results of his work entitled "The movement of the body immersed in the flow of radiant energy" were published in [1].

The scientific interest in the widespread use in astronomy of thread suspended instruments arose after the French scientist Maurice Allais discovered deviation of the swing plane of a paraconical pendulum (a kind of a Foucault pendulum on a short, rigid suspension) at the moment of a total solar eclipse 06.30.1954 [2].

Around the same time, a Leningrad professor N.A. Kozyrev used another thread suspended device (the so-called asymmetrical torsion balance) for observation of certain astronomical phenomena. This sensitive instrument responds to some close irreversible processes as evaporation of acetone, flame of a candle or a gas burner, overturning a hourglass, the presence of living objects and others [3] [4]. The Kozyrev's observations of astronomical phenomena by using a torsion balance mounted at the focus of a telescope-reflector are of particular interest. The asymmetric torsion balance seemed to be able to register the influence of certain astronomical objects, an unknown radiation of which rotating a beam of the torsion balance.

The works of the ternary mentioned scientists stimulated further research on the effects of environmental conditions on the moving parts of thread suspended instruments: Foucault's pendulums, paraconical pendulums, tiltmeters, torsion pendulums and torsion balances [5]-[11]. A more complete set of publications on this topic can be found in [12].

Thus objectively, by different researchers, at different times, by different instruments a certain radiation (field?) of the environment has been recorded which transfers a torque.

This radiation (field) causes rotation of a torsion balance arm-pointer, twisting of torsion pendulums, the deviation in the position of the suspended tiltmeters, rotation of a Foucault's pendulum and a paraconical pendulum oscillation plane.

Presumably a source of such radiation is the Sun since all reported cases of abnormal behavior of the thread suspended devices were observed during solar eclipses or at other moments of syzygy, when several celestial bodies are arranged in a straight line. Torsind is no exception. It also responds to the phenomena one way or another associated with the Sun: sunrises and sunsets, solar and lunar eclipses, transits of planets and conjunctions.

\section{Summary of the Equipment and Methods of Observation}

A natural consequence of the thread-suspended instruments evolution was the creation of an automatic torsion balance of improved design. The so-called TORSIND (torsion indicator) is a disc torsion balance on a silk suspension.

A simplified scheme of Torsind is shown in Figure 1. It is a disc of aluminum foil (paper is also acceptable) suspended horizontally with a filaments extracted from a cocoon of a silkworm. The movable part of the device is enclosed in a sealed glass housing. A WEB camera observes the rotation of the disc through the upper glass cover and transfers information to a computer.

The replacement of an arm-pointer in asymmetrical torsion balance by a symmetrical, very lightweight aluminum or paper disc (the material is not important) allowed to reduce the effect of air convection inside an enclosure to a negligibly small value.

Then a small mass of the disk (not more than $100 \mathrm{mg}$ ) provided very high sensitivity of the device. In addition, the use of monofilament from a cocoon of a silkworm gave to the device a feature not found in other torsion pendulums and balances.

The torsind's disc can slowly rotate in the same direction for a long time and thus the thread does not accumulate any reversed torque. This feature allows the device to record lengthy signals of small amplitude.

A detailed description of device is given in the papers [13] [14].

Two identical in construction working units of torsind (WEB_1 and WEB_2) were in service in an isolated tower of an idle telescope of the Main Astronomical Observatory in Kiev for 2010-2014.

The window in the laboratory room was closed and curtained with a black, opaque paper and the entrance to the room was not available to outsiders. 


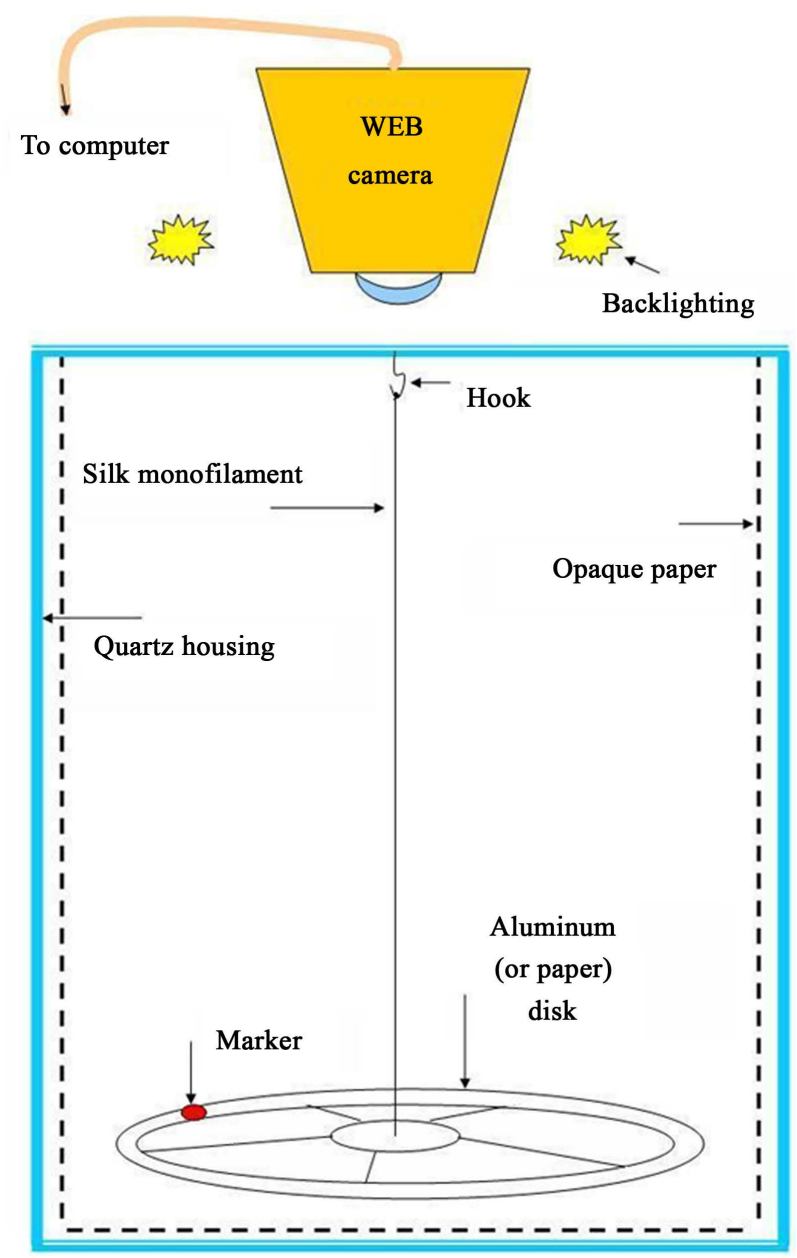

Figure 1. A schematic description of the torsind.

Throughout the whole working period the both units are not subjected to alterations, changes, they remained unchanged in their positions being located within a light-tight cardboard box. Never a ray of direct sunlight has fallen on the devices. There were no other buildings around the telescope tower at a distance of $50-100 \mathrm{~m}$ and no working electrical and electromechanical devices (except the computer), vibration, noise, and other noise sources.

There was no need for the presence of an operator, since the devices operated automatically, and the results were recorded in the computer memory in one-minute intervals.

\section{Special Torsind Features}

Our results of long-term measurements showed high reliability and efficiency of the torsind. Observations of several solar and lunar eclipses [11]-[19] allowed reliably to separate the basic phenomena associated with syzygy effects, apart from the background noise and fluctuations.

Herewith the three solar eclipses in 2009, 2010 and 2011 showed some common recurring features [11]. This happened despite the fact that all eclipses are specific and occurs in different conditions. This fact indicates that the results of the torsind measurements are free of significant errors, inaccuracies or instrument fluctuations and reliably reflect the strictly deterministic process. Our rationale for saying this is as follows.

1) A striking feature of the torsind is its ability to respond to changes in the near-Earth environment associated with the change in the balance of solar radiation coming to the Earth. This property is manifested not only at times of solar and lunar eclipses. Torsind "feels" the sunrise and sunset, clear responds to the so-called daily cycle [20]. 
2) In addition, the torsind reacts to astro-space events of non electromagnetic nature. Thus, observations of solar eclipses on 01.06.2011 and 9-10.05.2013 were carried out in a salt mine at a depth of about 40 meters. The instruments were inside a large $\left(\sim 1 \mathrm{~km}^{3}\right)$ wet monoblock of $\mathrm{NaCl}$ and reliably protected from any external electromagnetic influences, except for the hard cosmic rays. Nevertheless, the devices clearly reacted to eclipses [11] [19]. And their responses correlated with the reaction of the Foucault pendulum installed in the same place, and a paraconical pendulum mounted on the surface of the Earth.

This fact confirms the objectivity, accuracy and reliability of the torsind measurements.

3) Changes in gravitational potential does not affect the torsind readings. It is shown in [13] [20].

4) Due to its high sensitivity the torsind was able to register a previously unknown in science phenomenonspikes. Spikes are bursts of a not specified space radiation, which contains a torque.

This radiation causes the torsind disk to rotate. During a spike the torsind disk can do 3, 5, 10, 20, 30 or more consecutive rotations in one direction or another. The spikes are recorded over the past 5 years, with the spikes frequency and the average amplitude increasing [20] [21].

This increase agrees qualitatively with the simultaneous growth in solar activity. This fact also confirms the assumption that origin of the radiation which forces the torsind's disc to rotate, is associated with the Sun.

Thus, the device above described responds to the highly penetrating non-electromagnetic, non-gravitational radiation in nature, presumably associated with the Sun which comprises a torque spinning the mechanical system.

The brief above description of the torsind features is necessary for an adequate perception and understanding of some Venus observations presented below.

\section{Torsind Observations of Venus}

The following briefly describes the torsind reaction to the Moon-Venus conjunction on May 16, 2010 (when the two celestial objects had the same right ascension), and the transit of Venus on 5-6 June, 2012.

1) Conjunction with the moon

The observations were made during the three days (15, 16 and 17 of May). Three devices were involved in the observation process: two torsinds (WEB_1 and WEB_2), and an asymmetric beam torsion balance with a continuous results recording through a TV-camera (KV-TV).

The last unit was located in the neighboring building at a distance of about $150 \mathrm{~m}$ from the torsinds. Nobody came into the room, where there were instruments, throughout the period of the measurement. The result of the observations is shown in Figure 2. The three waves are imposed upon a descending trend, with maximum of the middle wave coinciding with the calculated moment of the conjunction. The vertical arrow indicates the moment of the Venus-Moon conjunction in right ascension. All three devices showed a similar picture. A significant response of the instruments started by $7 \mathrm{~h} 32 \mathrm{~m}$ before the conjunction and ended $4 \mathrm{~h} 56 \mathrm{~m}$ after.

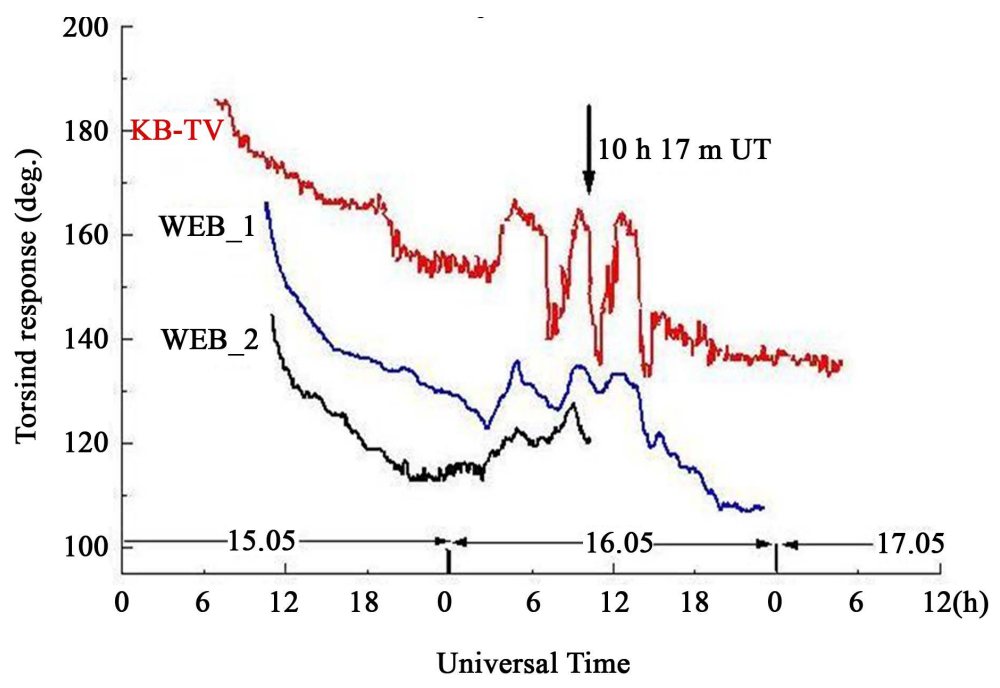

Figure 2. Venus/Moon conjunction on 16/05/2010. 
It is noteworthy that the correlation coefficient between the curves WEB_1 and KV-TV was quite high and amounted to 0.872 . This suggests unquestionable reliability of the result obtained.

2) Transit of Venus across the Sun's disc

Venus is the inner planet of the solar system. So sometimes this planet may stay on a line connecting the centers of the Sun and the Earth. At such moments, we are witnessing a phenomenon called "a transit".

The observations were made during 5, 6 and 7 of June with the devices WEB_2 and WEB_3. The apparent path of the planet across the solar disk and the transit circumstances are shown in Figure 3. The result of three days observations, which began one day before the transit, and terminated the next day after the end of the phenomenon, is shown in Figure 4. On 5th of June devices were registering only downward trend while the most significant reaction appeared in the morning of June 6 . The beginning of the phenomenon (the first contact- $\mathrm{I}$ ) and its completion (the last contact-IV) are indicated by dashed vertical lines. Both the devices showed a clear, mutually correlated response to the transit. Initially both the discs rotated clockwise, and then did in the opposite direction.

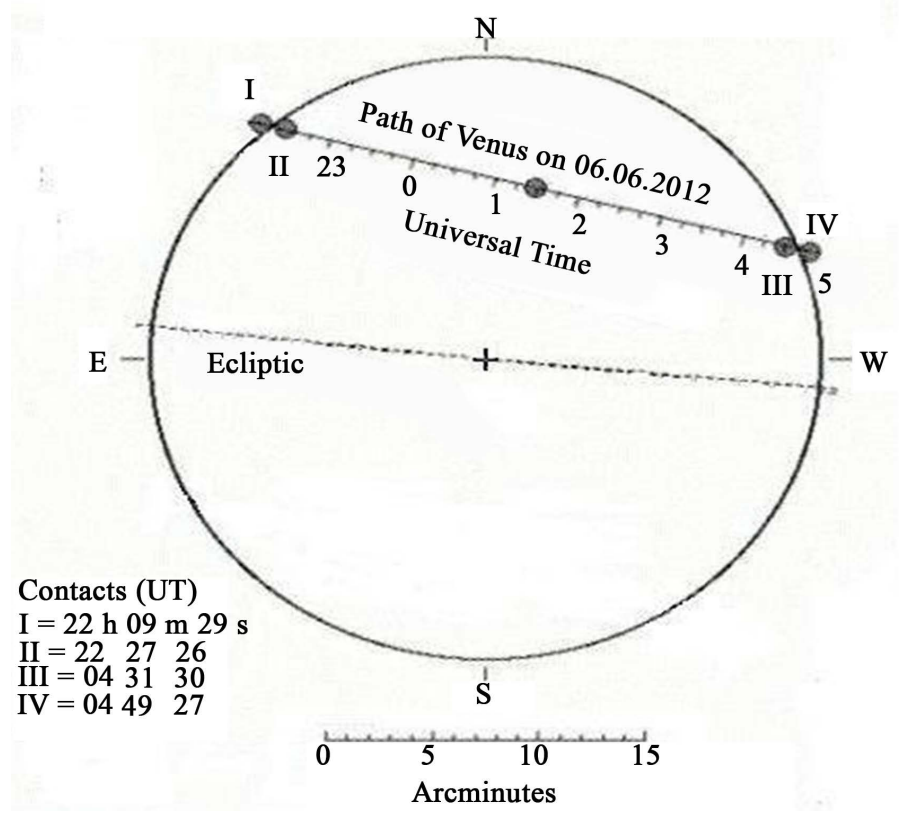

Figure 3. Transit of Venus 2012, June 06.

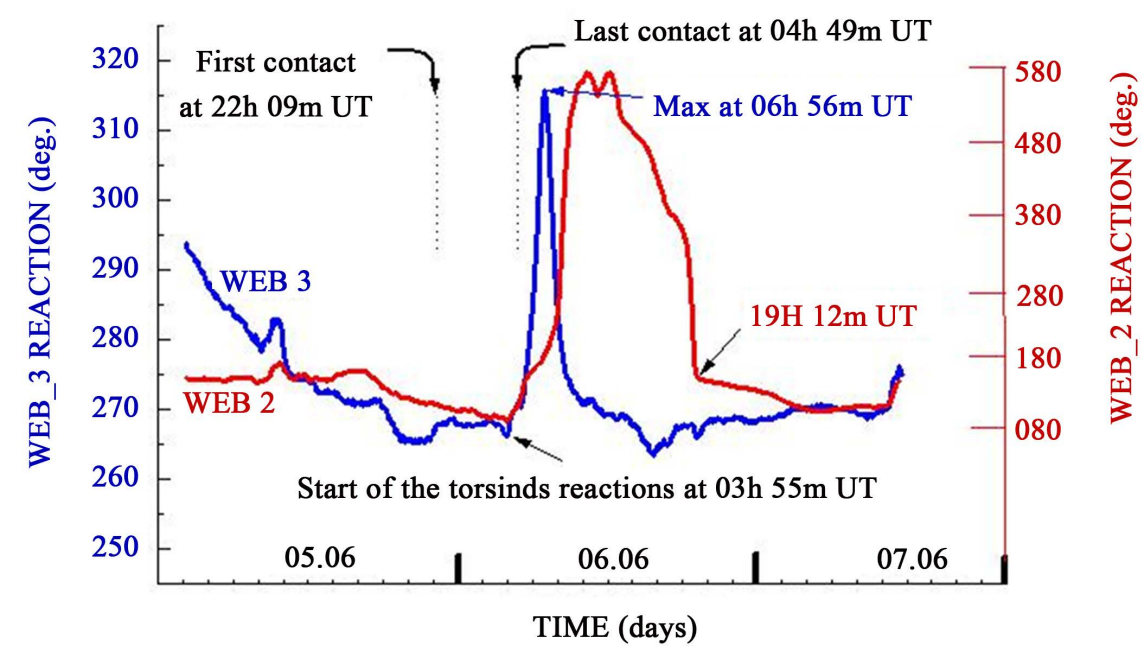

Figure 4. Venus transit on 5-6th of June, 2012. 
It is important that most of the time, while the projection of Venus crossed the sun's disk, there was no significant reaction of both instruments except for small fluctuations. After only $5 \mathrm{~h} 46 \mathrm{~m}$ behind the first contact both the devices began to increase their readings simultaneously. However, the reaction each of them was quite specific, as can be seen from the Figure 4. Obviously, that the reaction time, amplitude and arrival times of the maximum were different for each graph.

Thus, important results of these observations were that, firstly, both the devices similarly reacted on astronomical phenomenon and, secondly, that the reaction of both torsinds lagged behind the real events by nearly 6 hours.

We assume that the effect shown in Figure 3 was due to the deflection of the SVR flow in the gravitational field of Venus. The theory does not limit this effect.

A simplified diagram of the effect is shown in Figure 5.

This is a very simplified diagram. No complete analogy with an optical focusing effect exists. Firstly, there is no the focus point. Secondly, the trajectories of individual spirino are not straight lines.

Third, the gravitational field of Venus bends a spirino trajectory in another manner as an optical lens changes a direction of photons movement. Figure 4 shows only that the gravitational field of Venus can, under certain conditions, increase the flux of the SVSR near the Earth. It is possible that the torsind registers this effect.

\section{Discussion and Analysis}

\subsection{Properties of the Spiral Vortex Solar Radiation}

For the reasons stated in Section 3 it is hardly possible to interpret the result of these observations, being based on the orthodox doctrine of the four fundamental interactions. We need a fresh look at things. A radically new understanding of the facts can be helped with a radical departure from conventional practice.

One of new hypotheses has been proposed by the co-author of this work (N.G.A) and its essence is as follows. The hypothesis is based on the postulate that all irreversible processes in nature generate a spiral vortex field. Similarly, as photons are carriers of electromagnetic field, the carriers of the spiral vortex field are hypothetical spirino particles. The mass of a spirino is $1.2 \times 10^{-29} \mathrm{~g}$, the charge is zero, and the spin is \pm 1 . A stream of the spirino produces the spiral vortex radiation (SVR).

It is known that the main irreversible process throughout the Solar System is the fusion process of nucleosynthesis in the Sun's core. On this basis, it is concluded that the main source of spirino and SVR is the Sun. The spiral vortex solar radiation can occur on the surface of the Sun (background threads such as rain), in the sunspots in the form of collimated and coherent streams, as well as in chromospheric spicules.

For this reason, power characteristics of Sun's SVR from different sources differ significantly from each other and the energy spectrum is very wide. The propagation velocity of the Sun's SVR depends on the density of the space and the mass of the interplanetary substance being overcome on the SVSR path. Importantly, the SVR of the Sun carries a torque and is capable to transmit an angular momentum while interacting with a substance.

The above characteristics of spirino and SVR of the Sun are published in some theoretical works of Nikolsky et al. [22]-[26], to which an interested reader is referred.

\subsection{Observational Confirmations for the SVRS Hypothesis}

1) The most significant experimental confirmation of the plausibility of the SVR hypothesis is that the observation of the 06.06.2012 Venus transit allowed us to determine the velocity of the SVSR.

Gravitation Lensing of the Sun's SVR by Venus
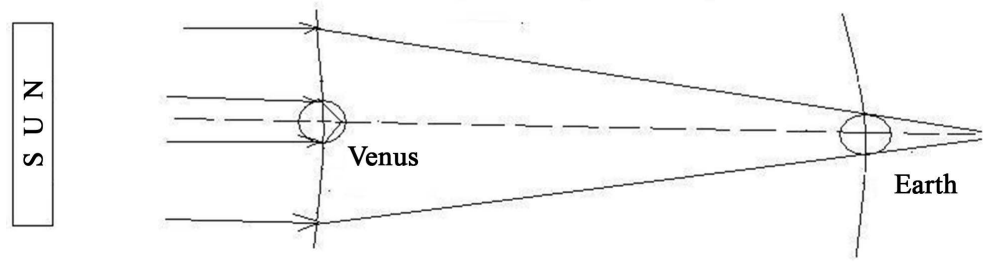

Figure 5. Gravitation lensing of the Sun's SVR by Venus. 
Earlier, Nikolsky presented a theoretical calculation of the spirino speed at different distances from the solar nucleus to the Earth [27]. According to the theory the spirino speed in the solar core centre is close to the speed of light; in solar spicules it is close to $3000 \mathrm{~km} / \mathrm{s}$, and at the outer border of the Sun-Earth radius it falls to the value of about $1000 \mathrm{~km} / \mathrm{s}$.

The first experimental determination of the spirino speed was made by Nikolsky in 2007. He used data from the literature [28] referring to the change in weights of the certain minerals samples in the days of high solar activity, 6-7 October 1993. A comparison between the time of passage of the active regions through the solar meridian and moments of change in weight of these minerals, give the SVR speed of $1740 \mathrm{~km} / \mathrm{s}$ [23].

Now, a more accurate value of the speed can be obtained from our torsind observations of the Venus transit.

It was noted above that the signals being registered by the torsinds were delayed relative to the moment of the first contact by $5 \mathrm{~h} 46 \mathrm{~m}$. If we assume that this delay was caused by the finite propagation speed of the SVSR signal from Venus to Earth, given the interplanetary distance 41.4 million $\mathrm{km}$ at the time of the transit yields that the SVR speed in the segment Venus-Earth amounted to

$$
\mathrm{V}_{\mathrm{SVR}}=1990 \mathrm{~km} / \mathrm{s}
$$

Thus, three different methods of determining of the SVR speed: theoretical (3000 km/s up to $1000 \mathrm{~km} / \mathrm{s}$ ); by geological samples $(1740 \mathrm{~km} / \mathrm{s})$; and from the delay of the torsind reaction $(1990 \mathrm{~km} / \mathrm{s})$ showed a reasonable concurrency. This coherency of the results reinforces the credibility of the SVR hypothesis under consideration.

2) The three-hour series of periodic oscillations of relatively small amplitude (from 0 to about 25 degrees) with a period of about 3 minutes was recorded on December 25, 2012. This episode is graphically shown in Figure 6.

It is noteworthy that for 4.5 hours before the appearance of the periodic oscillations and for 5 hours after their termination the torsind behaved very calmly. At this time, the device registered small fluctuations with an average amplitude of 3 - 5 degrees only.

A rigorous mathematical analysis of these observations showed that as the amplitude of the oscillations decreases, their frequency increased almost monotonically from $0.005379 \mathrm{~Hz}$ to a value of $0.005939 \mathrm{~Hz}$, which corresponds to a decrease in the period from 185.91s to 168.38s [29].

This event can be explained as an appearance of a single pulse of the SVR with a characteristic magnetoacoustic modulation. The theory predicts the emergence of such solitons. In this case standing waves with periods $300 \mathrm{~s}$ and $180 \mathrm{~s}$ are more likely to occur as compared with the oscillations of other periods. On 24-25 of December, 2012 the active region AO 1635 with a pronounced structural anomaly in the form of a compact group of several bright flames closely surrounding two main spots of the active region passed through the central meridian of the solar disk (see Figure 7).

This figure shows the visible hemisphere of the Sun on the date of 25.12.2012. Here, a compact group of the spots is visible near the meridian, designated as the active region AR 1635. On other Sun images in different wavelengths a dense group of bright faculae is visible on this position. We believe that appearance of the three-

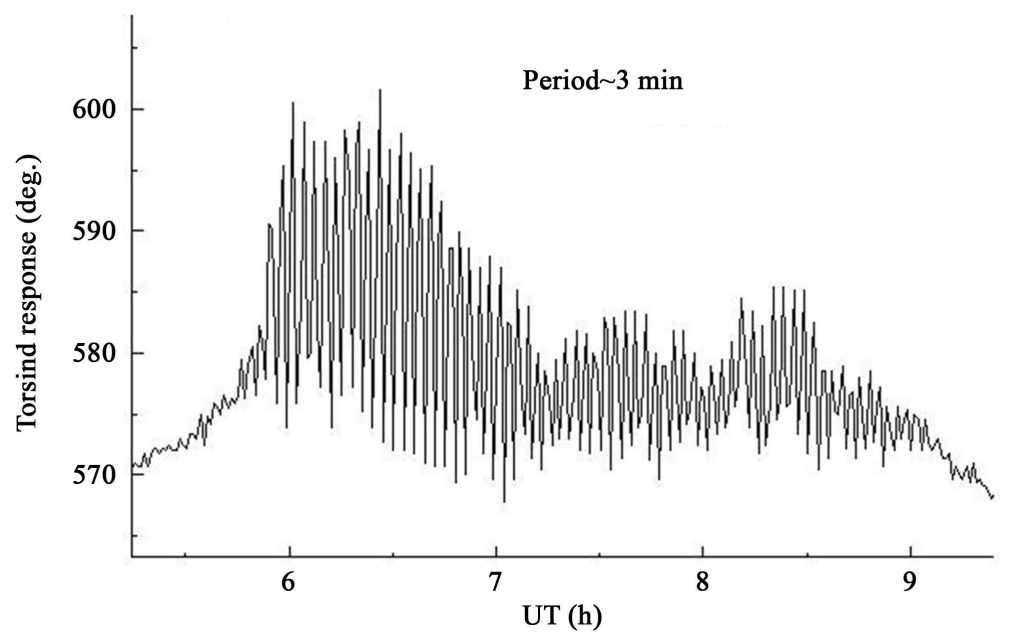

Figure 6. Periodic oscillations on 25.12.2012. 


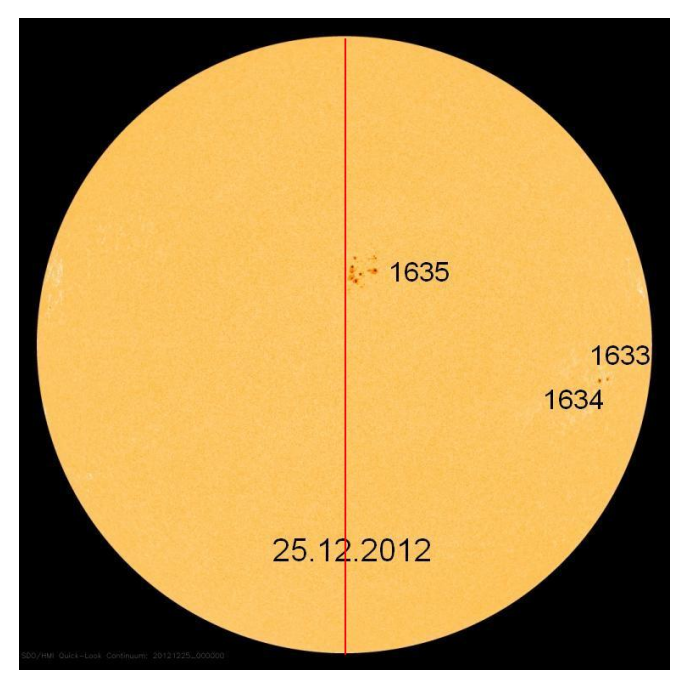

Figure 7. The location of the sunspots group 1635 relative to the solar meridian (in red) on the date of observation.

minute oscillations (Figure 6) was caused by the passage of the group AR 1635 through the solar meridian. Here are our arguments.

The active region AR 1635 crossed the Sun's meridian around noon on $24^{\text {th }}$ of December. The brightest spot in this group (the West) crossed the solar meridian at about $13.5 \mathrm{~h}$ (UT) on $24^{\text {th }}$ of December. Whilst our unit has registered the maximum of the three-minute oscillations at about 06.0 h UT on December 25.

It takes approximately 16.5 hours between these two events. Taking the distance to the Sun near perihelion equal to $147.1 \times 10^{6} \mathrm{~km}$, it is possible to determine the interaction transmission velocity from the star to the planet, which is equal to

$$
\mathrm{V}_{\text {Sun }}=2470 \mathrm{~km} / \mathrm{s}
$$

This value is slightly higher than the value $\mathbf{V}_{\text {SVR }}$ previously defined. The reason for this excess has a simple explanation. As previously indicated, the SVR speed along the Sun-Earth radius varies and is not the same. Near the Sun the speed is higher than in the vicinity of Venus or the Earth. That is why the average SVR speed on the Sun-Earth radius was slightly higher than in the neighborhood of Venus-Earth.

Thus, it is possible to draw 3 conclusions from the considerations mentioned above.

A) Two events - the transit of the region AR 1635 across the solar meridian and the appearance of the 3minute oscillations are likely causally related.

B) An additional confirmation was received that the SVR speed in the Earth-Venus sector is really close to the value of about $2000 \mathrm{~km} / \mathrm{s}$.

C) Excess of $V_{\text {Sun }}$ over $V_{\text {SVR }}$ proves the correctness of the theoretical conclusions that SVR speed in the interplanetary space is not constant and decreases along the Sun-Earth radius.

Taken together, these findings strongly favor the hypothesis on existence of the vortex solar spirino.

3) Spirino mass is 73 times less than the mass of the electron. The fact that spirino has a mass at rest confirms its origin in the course of disintegration processes in the region of nucleosynthesis within the Sun's core.

4) Since the spirinoes can be generated in different layers of the solar photosphere and chromosphere, they have different energies, i.e. solar vortex radiation flux has a wide energy spectrum. This fact is indirectly confirmed by our observations that torsinds with different response functions (in other words-with different "spectral" sensitivity) may respond differently to the same event.

For example, the similar, but not identical torsinds WEB-2 and WEB-3 showed a similar, but not identical reaction during the Venus transit (see Figure 3).

5) Spirino has no an electric charge, as indicated by the theory, and this feature provides them with a high penetrating power.

During the underground observations of the solar eclipse on 01.06 .2011 we saw that the torsind reliably registered SVR flow of the Sun, being in the thick of minerals at a depth of $40 \mathrm{~m}$. In addition, this fact is also an 
additional argument that the torsind responded precisely to the solar spirino, because its reaction was usually noted at the moment of the Solar event.

6) And finally, the most important thing. Flow of the SVSR forces the torsind's disc to rotate. This fact is recorded almost continuously for many years, and it can neither be denied nor refuted. Under the action of the SVSR the disc of the device rotates in one or other direction, indicating the presence of the vortex or torsional component in the Sun's radiation and in addition this component changes sign, since the rotation may occur in opposite directions as can be seen in numerous examples.

During the Venus transit the torsind's behavior can be explained as follows. As an example, we may consider the reaction of the device WEB_2, shown in Figure 3. Before starting of the phenomenon the WEB_2 readings were in the range of 080-180 degrees. This is a certain background value, which corresponds to the quasi-equilibrium state of right-handed and left-handed spirinoes in the solar radiation.

Once Venus appeared over the disc of the Sun, then the lensing mechanism has started to work, and rightspirino flux density increased in the Earth's direction. Since a pre-existing equilibrium has been broken the torsind WEB_2 responded to this event by turning the disc clockwise to the value of 540 degrees. After the transit has terminated and the lensing process ended off, the flux density of right-handed spirino decreased to the quasiequilibrium state. Therefore, after the end of the transit the instrument readings back exactly to this quasi-equilibrium level of 080-180 degrees, and remained so for another day. That is, the lensing effect was reflected in the fact that the flow density of a right-handed spirinoes has increased in the vicinity of Earth. Therefore, we can assume that the spirino stream coming from the northern hemisphere of the Sun contains more just a righthanded spirinoes.

This assumption is quite amenable to an experimental verification in the future. To do this, it is need to observe a transit of Venus or other celestial body from one hemisphere of the Sun to another one through the solar equator. According to our assumptions, at this time the polarity of the spirino flow should change.

\section{Conclusions}

The Venus transit study using the torsind allowed us to tie in a narrow knot a set of facts which help to understand the rotation reason of the torsind's disk. These facts indicate the presence of previously unexplored space radiation component of non-electromagnetic nature, which carries a torque. This torque, previously titled T-momentum [20], may be perceived by mechanical systems.

We have suggested that all irreversible processes are accompanied by generation of SVR. Since the main focus of irreversible processes in the solar system is the solar core where various nucleosynthesis reactions take place, the Sun is the main generator of SVR in the surrounding space.

Indirectly, this conclusion is confirmed by the fact that all astronomical phenomena to which the torsind responds are anyway associated with the Sun: sunrises and sunsets, solar and lunar eclipses, transits of planets and conjunctions.

The Venus transit on 06.06.2012 showed that solar spirino interacts with the gravitational field of the planet, and is deviated by them. As a result, such focused SVR flux entering the Earth varies. This change is recorded by the torsind.

If this is the case, then the other celestial bodies could cause the lensing effect when crossing the solar disk as seen from the Earth. Then this assumption is amenable to direct verification by torsind observations at the moments of transit or very close conjunctions. In particular, 08.11.2006 we observed a transit of Mercury across the solar disk using a conventional (arrow) torsion balance. Faint but clear positive effect was observed [17].

We assume that SVSR may be an observable manifestation of a new fundamental interaction. The basis for this assumption is the fact that the torsind used is not sensitive to changes in electromagnetic field. It was established during the underground observations of solar eclipses [11] [19]. Moreover, the torsind is not sensitive to changes in gravitational potential by definition. The design of such a balance makes it insensitive to variations in gravitational potential and ensures that it is unaffected by gravitational (tidal) influences from any direction. If it were not so, then the torsind should have been able to register a tidal wave from the Moon with the period of 12.3 hours [13]. This phenomenon was not observed, however.

These facts give us reason to believe that the Solar Vortex Radiation from the Sun can be an observable manifestation of a new fundamental interaction. 


\section{Competing Interests}

Authors have declared that no competing interests exist.

\section{Authors' Contributions}

The first author proposed the concept of SVSR, suggested the possibility of gravitational lensing and calculated a theoretical application. The second author manufactured the equipment, performed the observations and prepared the manuscript. The analysis and the conclusions were obtained together. All authors read and approved the final manuscript.

\section{References}

[1] Myshkin, N.P. (1906) Movement of a Body Located in the Flow of Radiant Energy. Russian Journal of Physical and Chemical Society, 3, 151-184. (In Russian)

[2] Allais, M.F.C. (1957) Movements of the Paraconical Pendulum and the Total Solar Eclipse of 30 June 1954. Proceedings of the French Academy of Sciences, Paris, 245.

[3] Kozyrev, N.A. (1991) Selected Works. Publ. Leningrad State University. (In Russian)

[4] Kozyrev, N.A. (1977) Astronomical Observations by Means of Physical Properties of Time. Proceedings of the Symposium, Dedicated to the Opening of the $2.6 \mathrm{~m}$ Telescope of the Byurakan Astrophysical Observatory, AN ArmSSR, Yerevan, 5-8 October 1976. (In Russian)

[5] Savrov, L.A. (2009) Improved Determination of Variation of Rate of Rotation of Oscillation Plane of a Paraconic Pendulum during the Solar Eclipse in Mexico on July 11, 1991. Measurement Techniques, 52, 339-343. http://dx.doi.org/10.1007/s11018-009-9291-6

[6] Savrov, L.A. (1997) Experiment with Paraconic Pendulums during the November 3, 1994 Solar Eclipse in Brazil. Measurement Techniques, 40, 511-516. http://dx.doi.org/10.1007/BF02504372

[7] Luo, J., Li, J.G., Zhang, X.R., Liakhovets, V.V., Lomonosov, M. and Ragyn, A. (1991) Observation of 1990 Solar Eclipse by a Torsion Pendulum. Physical Review D, 44, 2611-2613. http://dx.doi.org/10.1103/PhysRevD.44.2611

[8] Kuusela, T. (1991) Effect of the Solar Eclipse on the Period of a Torsion Pendulum. Physical Review D, 43, 20412043. http://dx.doi.org/10.1103/PhysRevD.43.2041

[9] Kuusela, T. (1992) New Measurements with a Torsion Pendulum during the Solar Eclipse. General Relativity and Gravitation, 4, 543-550. http://dx.doi.org/10.1007/BF00760136

[10] Saxl, E.J. and Allen, M. (1971) 1970 Solar Eclipse as “Seen” by a Torsion Pendulum. Physical Review D, 3, 823-825. http://dx.doi.org/10.1103/PhysRevD.3.823

[11] Olenici, D. and Pugach, A.F. (2012) Precise Underground Observations of the Partial Solar Eclipse of 1 June 2011 Using a Foucault Pendulum and a Very Light Torsion Balance. International Journal of Astronomy and Astrophysics, 2, 204-209. http://dx.doi.org/10.4236/ijaa.2012.24026

[12] Pugach, A.F. (2011) Should the Laws of Gravitation Be Reconsidered? Apeiron, Montreal.

[13] Pugach, A.F. (2013) The Torsind-A Device Based on a New Principle for Non-Conventional Astronomical Observations. International Journal of Astronomy and Astrophysics, 3, 33-38. http://dx.doi.org/10.4236/ijaa.2013.32A006

[14] Pugach, A.F. (2014) Torsind Is the Device of the New Physics. International Journal of Unconventional Science, 2, 6-13 (In Russian)

[15] Pugach, A.F., Shatokhina, S.V., Lazorenko, P.F., Karbovsky, V.L., Medvedsky, M.M., Lazorenko, G.A., et al. (2008) The First Experience of Solar Eclipse Observations with a Miniature Torsion Balance. Kinematics and Physics of Celestial Bodies, 24, 253-258. http://dx.doi.org/10.3103/S088459130805005X

[16] Goodey, T.J., Pugach, A.F. and Olenici, D. (2010) Correlated Anomalous Effects Observed during the August 1 st 2008 Solar Eclipse. Journal of Advanced Research in Physics, 1, 021007.

[17] Pugach, A.F. (2009) Observations of the Astronomical Phenomena by Torsion Balance. Physics of Consciousness and Life. Cosmology and Astrophysics, 9, 30-51.

[18] Pugach, A.F. and Olenici, D. (2012) Observations of Correlated Behavior of Two Light Torsion Balances and a Paraconical Pendulum in Separate Locations during the Solar Eclipse of January 26th, 2009. Advances in Astronomy, 2012, Article ID: 263818. http://dx.doi.org/10.1155/2012/263818

[19] Olenici, D., Pugach, A.F., Cosovanu, I., Lesanu, C., Deloly, J.-B., Vorobyov, D., et al. (2014) Syzygy Effects Studies Performed Simultaneously with Foucault Pendulums and Torsinds during the Solar Eclipses of 13 November 2012 and 10 May 2013. International Journal of Astronomy and Astrophysics, 4, 39-53. 
http://dx.doi.org/10.4236/ijaa.2014.41006

[20] Pugach, A.F. (2015) Diurnal Variations and Spikes by the Torsind Registered and Their Impact on the Accuracy of G Measurement. International Journal of Astronomy and Astrophysics, 5, 28-37. http://dx.doi.org/10.4236/ijaa.2015.51005

[21] Pugach, A.F. (2013) Torsind as a Recorder of a Possibly New Energy. Thermal Energy and Power Engineering, 2, 129-133.

[22] Nikolsky, G.A. (2015) On Large-Scale Interactions of the Main Physical Fields. New on the Impact of the Sun on the Environment. Lambert Academic Publishing, Saarbrucken, 28-44. (In Russian)

[23] Nikolsky, G.A. and Pugach, A.F. (2015) On Determination of Component of the Solar Vortex Field: The Reason, Ensured the Existence of the Civilization. Lambert Academic Publishing, Saarbrucken, 72-88. (In Russian)

[24] Nikolsky, G.A. (2012) The Most Likely Sources of the Microwave Vortex Radiation of the Sun: Effects of Their Impact on the Geosphere. Proceedings of the 1st Interregional Symposium "Ecology and Space”, St. Petersburg State University, 374-387. (In Russian)

[25] Nikolsky, G.A. and Pugach, A.F. (2012) Exclusive Sources of Power Penetrating Solar Radiation and the Transit of Venus. Proceedings of the 8th International Conference "Natural and Anthropogenic Aerosols", 194-214. (In Russian) http://vd2-777.narod.ru/article12/reason.htm

[26] Nikolsky, G.A. (2015) The Phenomenon of Gravitational Lensing of the Field of Sun's Penetrating Radiation by the Venus. New on the Impact of the Sun on the Environment. Lambert Academic Publishing, Saarbrucken, 89-117. (In Russian)

[27] Nikolsky, G.A. (2009) On the Fifth Interaction. (In Russian) http://vd2-777.narod.ru/files/GNikolsky_101017About_5th_interaction.doc

[28] Eganova, I.A., Samoilov, V.N., Struminsky, V.I. and Kallis, V. (2007) Certain Gravitation Problems and Research Opportunities in Geophysics Monitoring. Preprint of the Joint Institute for Nuclear Research, Dubna, No. P2-2007-183.

[29] Scholkmann, F. and Pugach, A.F. (2015) Unexplained Oscillations in Deflection Angle Fluctuations of a Novel Type of Torsion Balance. Progress in Physics, 11, 247-251.

\section{Submit or recommend next manuscript to OALib Journal and we will provide best service for you:}

- Publication frequency: Monthly

- 9 subject areas of science, technology and medicine

- Fair and rigorous peer-review system

- Fast publication process

- Article promotion in various social networking sites (LinkedIn, Facebook, Twitter, etc.)

- Maximum dissemination of your research work

Submit Your Paper Online: Click Here to Submit

Contact Us: service@oalib.com 